\title{
Decentralized Multiuser Cooperative Spectrum Sensing in Cognitive Radio System
}

\author{
Nasrullah Armi ${ }^{1}$, Mohd. Zuki Yusoff ${ }^{2}$, and N. M. Saad ${ }^{2}$ \\ ${ }^{1}$ Indonesian Institute of Sciences, Research Center \\ for Electronics and Telecommunication Bandung, Indonesia \\ ${ }^{2}$ Department of Electrical and Electronics Engineering, Universiti Teknologi PETRONAS \\ Bandar Seri Iskandar, Perak, Malaysia \\ ${ }^{1}$ nasrullah.armi@gmail.com
}

\begin{abstract}
Cooperative spectrum sensing (CSS) was proposed as one solution to overcome uncertainty noises in primary signal detection. It increases capability of primary signal detection and accuracy. This paper investigates the performance of decentralized CSS model with limited sensing capability in cognitive radio (CR) system. Simulation considers multiple secondary users (SUs) sense license channel and distributes their sensing result among others. Partially observable Markov decision process (POMDP) framework is used as an aid for SU that partially sense over license channel. It models license channel as Markov discrete process where idle and busy states is represented by number of " 0 " and "1". To validate this work, we make some comparisons against single user sensing, centralized multiuser cooperative spectrum sensing (CMCSS) and decentralized random CSS model. The derived results show that CMCSS and DMCSS model has better performance than single user sensing and decentralized random CSS model. CMCSS outperforms DMCSS model. However, DMCSS model still outperforms decentralized random CSS. At certain case, DMCSS performance matched to the throughput achieved by CMCSS when probability of selected channel $(p)=1$.
\end{abstract}

Keywords: Cognitive Radio (CR); Dynamic Spectrum Access (DSA); Opportunistic Spectrum Access (OSA); Partially Observable Markov Decision Process (POMDP); Decentralized Network

\section{Introduction}

Dynamic Spectrum Access (DSA) system is a new paradigm for spectrum allocation and one of the most promising technologies available for efficient spectrum use since the growth of wireless user number. It replaces static spectrum allocation and aims to increase the spectrum utilization [1]. Opportunistic spectrum access (OSA) is part of DSA systems which has a capability to identify unutilized spectrum and arrange which user can access by prior reconfiguration of its parameter, i.e. modulation, transmission power, etc. OSA system works on overlay channel spectrum, therefore it must be convinced that there is no interference to license user by prior sensing to the spectrum environment, as DARPA XG project or through pre-existing knowledge, such as the geolocation database proposed for unlicensed access to TV band white space, or a combination of both [2]. Shortly, OSA is one solution for spectrum scarcity due to the growth of wireless user number and has the benefits such as better spectrum management, dynamically unutilized spectrum access, spectrum sharing, etc.

Cognitive radio (CR) is an enabling technology for OSA system. It is a prospective and future technology to be implemented in spectrum access system as a replacement of static spectrum utilization rule. It has a capability to detect the spectrum holes or white space and dynamically access them by prior sensing. CR user that is also known as secondary user (SU) can share the available channel spectrum by arranging which user can access and when it must be performed. The cognitive MAC protocol manages the channel access strategy, which channel can be sensed and accessed by CR user based on sensing outcome and observation. This protocol design aims to achieve the Quality of Service (QoS) of data transmission. 
Cooperative spectrum sensing is defined as kind of primary signal detection technique where SU exchanges their local sensing result and observation among others. It is able to improve the fidelity of sensing outcome and as a solution of primary signal detection problem over fading, shadowing, hidden and exposed node, etc. Cooperative spectrum sensing was studied in [5]-[9]. However their studies based on the assumption that SU has the full band sensing capability. In fact, the cost to achieve wide band spectrum sensing by a single $\mathrm{SU}$ is quite high.

Cluster-based decentralized cooperative spectrum sensing network was proposed in [10]. Each node in a cluster decides which spectrum should be monitored according to the tracking history of spectrum availability. The authors claimed that the proposed algorithm reached the performance of centralized at a certain circumstance while outperforming the Round Robin scheme. Centralized and decentralized cooperative spectrum sensing is also discussed in [11]. The state of primary user traffic is described as a Hidden Markov Model to improve the performance of centralized and decentralized cooperative spectrum sensing.

To our knowledge so far, Q. Zhao et al [12][13] were among the earliest researchers who investigated the limited sensing capability of each SU in OSA under POMDP frameworks. They presented a cross layer design approach to investigate spectrum sensor point in physical layer and the access strategy to optimise the throughput performance in MAC layer. Then, in the following years, the other authors in [14]-[20] adopted POMDP frameworks to investigate dynamic spectrum access performance. However, they considered single user spectrum sensing. Multiuser spectrum sensing in decentralized OSA network was investigated in [21]. The authors assume that SU senses license spectrum without exchanging their sensing results on perfect channel condition.

In this paper, we investigate multiuser decentralized cooperative spectrum sensing under POMDP frameworks. SUs sense the spectrum availability and exchange their local sensing result. SUs decide which and when channels can be accessed without forwarding the sensing results to the central coordinator (decentralized cooperative sensing). The rest of the paper is organized as follows. In section II, we give description of problem formulation for sensing and access strategy. POMDP formulation is included to select a part of channel for sensing and access. The detail system model is described in section III. The numerical results are presented along with some comparisons in section IV. Finally, conclusion is presented in the following section.

\section{Spectrum Access Model under POMDP}

POMDP is an aid in the automated decision-making. POMDP policy informs SU on what action is to be executed. It can be a function or a mapping and typically depends upon the channel states. In this section, we provide formulation of spectrum sensing and POMDP framework for OSA system model.

\section{A. Spectrum Sensing on Physical Layer}

Spectrum sensing is performed by SU in the physical layer by taking number $k$ of channel measurement $Y_{n} \equiv\left[Y_{l}, \ldots, Y_{k}\right]$ from the chosen channel and performs binary hypothesis test as follows:

$$
\begin{aligned}
& H_{0}\left(S_{n}=0\right)=\text { idle }=Y_{n} \approx N\left(0_{k}, \sigma_{0}{ }^{2}\right) \\
& H_{1}\left(S_{n}=1\right)=\text { busy }=Y_{n} \approx N\left(0_{k}, \sigma_{1}{ }^{2}\right)
\end{aligned}
$$

where $N\left(0_{k}, \sigma_{0}{ }^{2}\right)$ denotes the $k$-dimensional Gaussian distribution with identical mean 0 and variance $\sigma^{2}$ in each dimension. The notation of $\sigma_{0}{ }^{2}$ and $\sigma_{1}{ }^{2}$ denote noise and primary signal power in channel $n$, respectively. A detector optimal under Newman and Pearson (NP) criteria is as follow [11]:

$$
\|Y\|^{2}=\sum_{i=1}^{k} Y_{i}^{2}{ }_{<H_{0}}^{>H_{1}} T
$$


The probability of miss-detection $\left(P_{M}\right)$ and the probability of false alarm $\left(P_{F}\right)$ are the following incomplete gamma function, which is given by [12] as follows:

$$
\begin{aligned}
& P_{M}=\delta_{n}=\gamma\left(\frac{k}{2}, \frac{T}{2\left(\sigma_{0}^{2}+\sigma_{1}^{2}\right)}\right) \\
& P_{F}=\varepsilon_{n}=1-\gamma\left(\frac{k}{2}, \frac{T}{2 \sigma_{0}^{2}}\right)
\end{aligned}
$$

where $\gamma(m, a)=\frac{1}{\Gamma(m)} \int_{0}^{a} t^{m-1} e^{-t} d t$ is the incomplete gamma function and $\mathrm{T}$ denotes a signal detection threshold. The receiver-operating curve (ROC) between false detection $(\varepsilon)$ and missidentification $(\delta)$ affects the optimal access strategy. The separation principle was presented in [6], in which the partition of the ROC curve is divided into conservative and aggressive access strategies. Hence, the optimal decision can be achieved when $\delta_{n}{ }^{*}=\zeta$, where $\zeta$ is maximum collision allowable by PU, that is known as collision threshold.

\section{B. POMDP Framework}

MAC layer in the OSA system has a responsibility to schedule which user and when SU can access the available channels. However, due to the limited sensing capability, SU only selects a part of channels to be sensed and accessed. POMDP framework models the channel spectrum opportunity as a Markov discrete process with a number of channel states. The change of states can be formulated as $\mathrm{M}=2^{\mathrm{N}}$ states, where $\mathrm{N}$ is number of channel. The state diagram for $\mathrm{N}=2$ is described in fig. 1 where $\bar{\alpha}_{l}=1-\alpha_{i}$ and state $(0,1)$ indicates the first channel is available and the second channel is busy. The term of partially observable means that SU selects a set of channels to be sensed and a set of channels to be accessed based on sensing outcome. This objective is to maximize the throughput of SU under the constraint of interference to PU by exploiting the sensing history and the spectrum occupancy statistics.

OSA protocol that maximizes the throughput of SU can be formulated as POMDP over finite horizon. It is defined by tuple $\{S, A, P, \Theta, R\}$, where $S$ denotes a finite set of states with state $\mathrm{i}$ denoted by $\mathrm{s}_{\mathrm{i}}$, A denotes a finite set of actions with action $\mathrm{i}$ denoted by $a_{i}, \mathrm{P}$ denotes the transition probabilities $p_{i, j}$ for each action in each state as a function of $\left\{\alpha_{i}, \beta_{i}\right\}_{i=1}^{N}$ which describes the channel availability of PU networks, R denotes the reward structure $\left(r_{j, A_{1}, A_{2}}\right)$ which is defined as the number of transmitted bits in one slot when the SU takes an action, and $\Theta$ is observation where SU observes the availability of channel at state $\mathrm{j}, \Theta_{j, A_{1}} \in\{0,1\}^{\left|A_{1}\right|}$. The reward is proportional to its bandwidth and it can be formulated as follows:

$$
r_{j, A_{1}, A_{2}}(t)=\sum_{i \in A_{2}} S_{i}(t) B_{i}
$$

Figure 2 shows the Markov dynamics process model for OSA system where observations are made after an action is taken. Equivalently, observation could have been taken before actions. The channel spectrum is sensed continuously even SU still access a certain channel and transmit data since it must vacate the band when PU returns back and use the spectrum. Based on the information state, SU has an option whether access the available channels or turn on sleep mode (no transmission due to the busy channel state).

In POMDP model, the system state is not directly known. However SU can observe to learn the most likely state. The observation yields the current system state. Then, the information state, also known as a belief vector $\pi=\left(\pi_{1}, \ldots, \pi_{M}\right)$, aids in determining the most likely state of the primary network by storing all previous actions and observations in a summary statistic. The belief vector is probability distribution over the state of the channels.

The belief vector $\pi$ is a sufficient statistic for the optimal policy and behaves as a discrete time continuous state Markov process. The users observe with distribution probability under the system channel states. The information state is updated after each action and observation, which has the transformation information as equation bellows:

$$
\pi^{\prime} \cong\left[\pi_{1}^{\prime}, \ldots, \pi_{M}^{\prime}\right] \cong \tau(\pi \mid a, \theta)
$$


Nasrullah Armi, et al.

$$
\begin{gathered}
V_{t}(\pi)=\max _{a=1, \ldots, N}\left\{\sum_{i=1}^{M} \pi_{i} \sum_{j=1}^{M} p_{i, j} \sum_{\theta=0}^{1} \operatorname{Pr}\left[\Theta_{j, a}=\theta\right]\left(\theta B_{a}+V_{t+1}(\tau(\pi \mid a, \theta))\right)\right\} \\
W_{t}(\Omega)=\left(\omega_{a *} \beta_{a *}+\left(1-\omega_{a *}\right) \alpha_{a *}\right) B_{a *}+\sum_{\theta=0}^{1} \operatorname{Pr}\left[\Theta_{a *}=\theta \mid \Omega, a_{*}\right] W_{t+1}\left(\tau\left(\Omega \mid a_{*}, \theta\right)\right) \\
=B_{a *}+\left[\omega_{a *}\left(1-\beta_{a *}\right)+\left(1-\omega_{a *}\right)\left(1-\alpha_{a *}\right)\right] W_{t+1}\left(\tau\left(\Omega \mid a_{*}, 0\right)\right)+ \\
{\left[\omega_{a *} \beta_{a *}+\left(1-\omega_{a *}\right) \alpha_{a *}\right] W_{t+1}\left(\tau\left(\Omega \mid a_{*}, 1\right)\right)}
\end{gathered}
$$

where $p_{i, j}$ denotes transition probability for each action in each state as function of $\left\{\alpha_{i}, \beta_{i}\right\}_{i=1}^{N}$ which describe the channel availability of PU networks; $B_{a}=$ channel bandwidth, $\Theta$ is observation for availability of channel state, $V_{t}(\pi)$ is the maximum expected reward that can be accrued in the remaining $\mathrm{t}$ decision intervals, and $W_{t}$ is the expected remaining reward starting from slot $t$ achieved by Greedy approach [16].

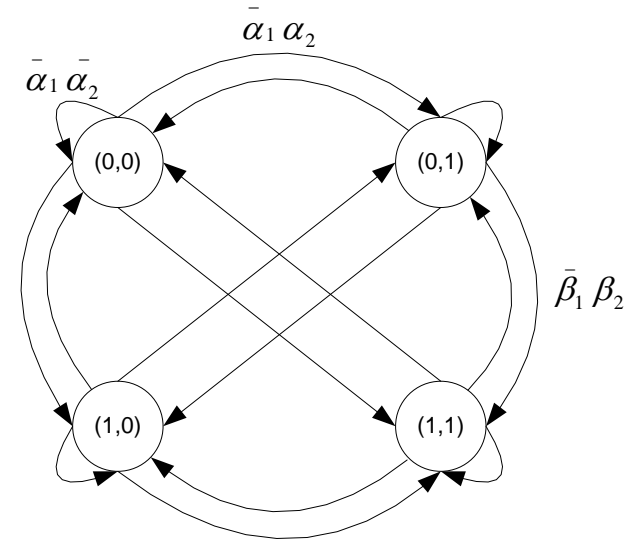

Figure 1. State diagram for $\mathrm{N}=2$ as Markov process model

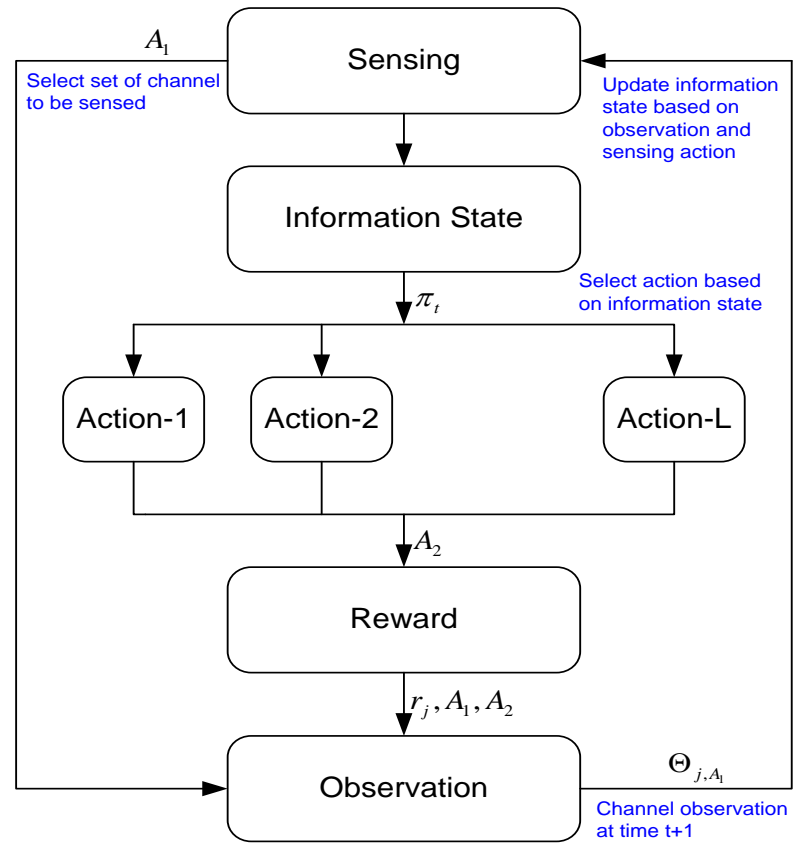

Figure. 2. OSA modelling under POMDP Frameworks 
In POMDP model, the policy maps the information states into action and maximizes the expected total reward. There are an infinite number of information states, since it is a probability distribution over all states. The policy or value function (i.e., information states) is stored by the SU in the form of tables. The maximum value function for all actions is given by the equation (8).

Due to the complexity of optimal strategy computation when number of slot and channel increase, Q. Zhao et al in [5] proposed the reduced sub-optimal strategy. They also presented that the performance of sub-optimal strategy relatively close to the optimal sensing policy. The recursive equation which is reduce the complexity based on Greedy policy is formulated as (9), where $\mathrm{Wt}(\Omega)$ is the expected remaining reward starting from slot $\mathrm{t}$ achieved by greedy approach, $\tau\left(\Omega \mid a_{*}, \theta\right)$ denotes the updated information of sensing outcome given the observation $\theta$ under action $a$. The notation of $a^{*}$ is defined as the selected action in slot $\mathrm{t}$ in order to maximize the expected immediate reward and given by

$$
a_{*}(t)=\operatorname{argmax}_{a=1, \ldots, N}\left(\omega_{a}(t) \beta_{a}+\left(1-\omega_{a}(t)\right) \alpha_{a}\right) B_{a}
$$

Fading, noise, and obstacles cannot be ignored in a wireless communication link. These imperfect conditions can cause some errors in PU signal detections and spectrum sensing. As presented previously, a false detection senses idle states as a busy channel and SU refrain from data transmission. On the other hand, miss-identification senses busy states as an idle channel and causes SU collide with the PU transmission. SU performs binary hypothesis where $\mathrm{H}_{0}=\mathrm{S}_{\mathrm{n}}(\mathrm{t})=0$ (idle) and $\mathrm{H}_{1}=\mathrm{S}_{\mathrm{n}}(\mathrm{t})=1$ (busy). Let $\Theta_{\mathrm{n}}(\mathrm{t}) \in\{0$ (idle), 1 (busy) $\}$ denote the sensing outcome of binary hypothesis. The performance of spectrum sensing is characterized by $P_{f a}$ $\left(\varepsilon_{\mathrm{n}}(\mathrm{t})\right), \mathrm{P}_{\mathrm{m}}\left(\delta_{\mathrm{n}}(\mathrm{t})\right)$. The channel $a^{*}$ selected for both optimal and greedy approaches is thus given by

$$
a_{*}(t)=\arg \max _{a=1, \ldots, N}\left(\omega_{a}(t) \beta_{a}+\left(1-\omega_{a}(t)\right) \alpha_{a}\right)(1-\varepsilon) B_{a}
$$

The belief vector should be updated both at the transmitter and receiver. Information at the transmitter in one slot includes the decision $\left(a_{*}, \Phi_{a_{*}}\right)$ and the observation $\left(\Theta_{\mathrm{a}^{*}}, \mathrm{~K}_{\mathrm{a}^{*}}\right)$ where $\mathrm{K}_{\mathrm{a}^{*}} \in\{0,1\}$ denotes an acknowledgement at the end of the slot. However, information at the receiver only covers $a^{*}$ and $K_{a *}$ since the receiver does not have the sensing outcome $\Theta_{a *}$ and cannot distinguish an unsuccessful transmission from no access decision $\Phi_{a_{*}}=0$.

\section{Cognitive Radio Network Model}

The spectrum containing a number of channels is licensed to PU who has the authority to them. However, when they are not used, SU can access the channels with prior observation whether channels are available or not in order to avoid interference to PU. We consider a group of SU who sense and monitor primary networks activity, which change depends on the time step and switch from occupied and unoccupied according to the Markov discrete chain. The existing channels are shared among PU and a large number of SUs.

There are number of channels considered in this study and state of these channels change independently. Each channel has the bandwidth $B i(i=1, \ldots, N)$. The state of channel $S_{n}(\mathrm{~T})=$ $\{1,0\}$ indicates that channel is busy and idle. In the system, the transmission time is divided into slots of equal length $\mathrm{T}$, where slot $k$ refers to the discrete time period [kT, $(\mathrm{k}+1) \mathrm{T}]$. At the beginning of each slot, SU sense set of the channels $\left(\mathrm{L}_{1}\right)$. Based on the sensing outcome, $\mathrm{SU}$ will decide which channel to be accessed $\left(\mathrm{L}_{2}\right)$, where $\mathrm{L}_{2} \leq \mathrm{L}_{1} \leq \mathrm{N}$. At the end of the slot, $\mathrm{SU}$ will send the acknowledgement signal that indicates successful transmission. The traffic statistics of the primary network follows a Markov discrete time process with number of states. Furthermore, secondary network is seeking spectrum opportunity in these $\mathrm{N}$ channels.

Single or multiple SUs sense either single or multiple channel availability. A single user (without cooperation) senses spectrum availability and access when it is unoccupied by PU. The SUs access the channel and transmit data based on their sensing outcome, perform sensing continuously during data transmission, and vacate the channel quickly when PU is detected. In 
multi user sensing, spectrum opportunities are sensed by multiple SUs. The users compete to sense an unoccupied channel and access it based on the sensing outcome.

Cooperative spectrum sensing among users has been proposed to overcome fading, shadowing, and uncertainty noises that occur in PUs detection. There are two kinds of cooperative spectrum sensing strategy namely centralized and decentralized cooperative sensing as described in Figure 3 and Figure 4.

As described in Figure. 3, each SU senses spectrum opportunities in CMCSS model and a central coordinator (CR base station) allocates one of the $M$ numbers of channels at the sensing stage. Then, at the report stage, each user forwards the sensing results to the central coordinator for final decision. The central coordinator decides which channels are idle and busy based on the sensing results and forwards it back to the users. The probability of detection $\left(C_{D}\right)$, probability of miss detection $\left(C_{M}\right)$, and probability of false alarm $\left(C_{F}\right)$ for CMCSS can be calculated by using the following formulas:

$$
\begin{aligned}
& C_{D}=1-\prod_{1}^{n}\left(1-P_{D, k}\right) \\
& C_{M}=1-C_{D}=\prod_{1}^{n}\left(1-P_{D, k}\right) \\
& C_{F}=1-\prod_{1}^{n}\left(1-P_{F, k}\right)
\end{aligned}
$$

Where $\mathrm{P}_{\mathrm{D}, \mathrm{k}}$ and $\mathrm{P}_{\mathrm{F}, \mathrm{k}}$ are the detection probability and false detection probability, respectively.

Subsequently, in DMCSS model, each user senses and selects channel without centralized coordinator. At the beginning of a slot t, SU selects the existing channel and perform local sensing. Then, each SU distributes local sensing cooperatively and exchanges the local observation among one another. They makes a final decision as to which channel can be accessed based on the sensing outcome. Data transmission is the following task to be performed when channels are available and SU receivers acknowledge the transmitter for successful data transmission. However, DMCSS cannot use Eq. (12) to (14) to derive local and cooperative miss detection probability. We assume that cognitive radio users $i$ select $m$ channels to sense with probability $\mathrm{P}_{\mathrm{i}, \mathrm{m}}$ at each time slot, and the expected miss detection probability for local sensing is $\mathrm{P}_{\mathrm{M}}$. According to the binomial distribution theory, the probability that users $i$ select $m$ channels can be calculated as below:

$$
\left(\begin{array}{l}
n \\
i
\end{array}\right) p^{i}(1-p)^{n-i} \quad i=1,2, \ldots, n
$$

where $n, i$, and $p$ denote number of $\mathrm{CR}$ user, number of user that select channel $m$, and probability of user $i$ to sense channel $m$, respectively. The average of miss detection probability, $C_{M}$, for distributed cooperative sensing result can be calculated as the following formula:

$$
\begin{aligned}
P_{M} & =\frac{\left(\zeta\left(1-(1-p)^{n}\right)+(1-p)^{n}\right)^{\frac{1}{n}}+(p-1)}{p} \\
C_{M} & =\frac{P_{M} x \text { Prob. of user select channel to sense }}{\sum_{i=1}^{n} \text { Prob.of user select channel }}
\end{aligned}
$$

where $\zeta$ denotes a collision threshold or a maximum collision probability allowed by PUs.

\section{Numerical Results and Discussion}

In this section, computer simulation results are presented to evaluate the performance of cooperative spectrum sensing strategy for OSA system under POMDP frameworks. The performance was evaluated based on the greedy sensing policy. Sensing errors are considered in this simulation as well, in which SU senses spectrum opportunities over imperfect channels. At the beginning of slot, multiple SUs sense the spectrum opportunities. For SUs who do not have packets to transmit, they do not need to participate in channel selection for sensing and turn sleep mode on. They keep updating their belief states according to the Markovian model of spectrum occupancy. SUs that have packets to transmit select channels and update their belief states based on sensing outcomes. If multiple SUs select one available channel, we assume that one of them will succeed in channel access and packet transmission. 


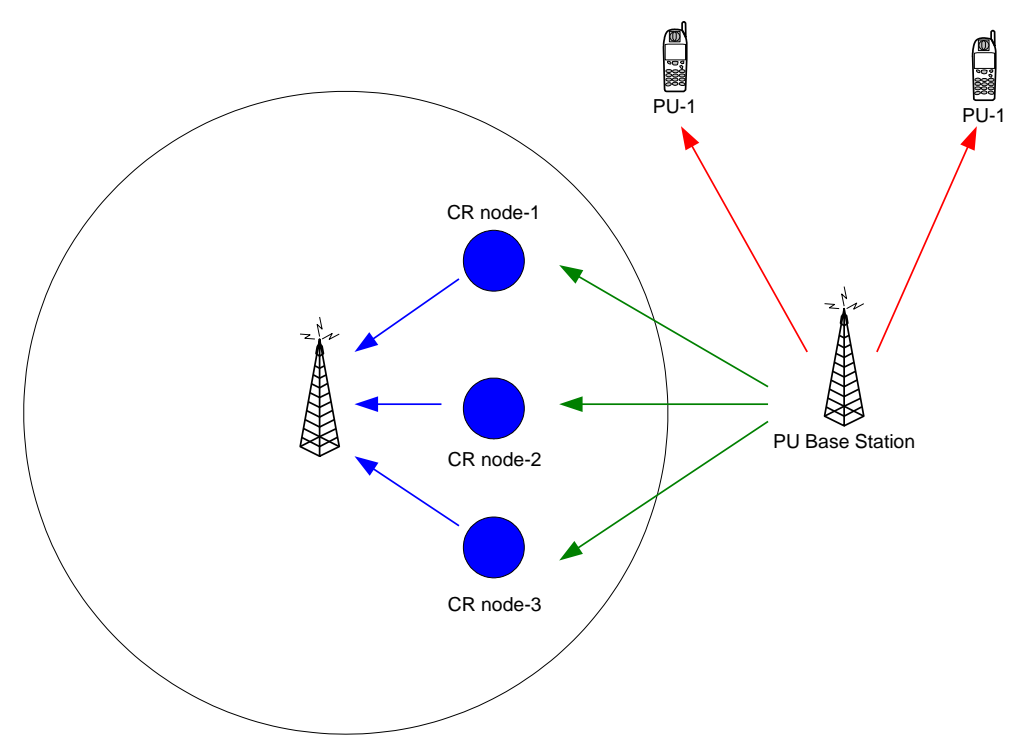

Figure 3. CMCSS model

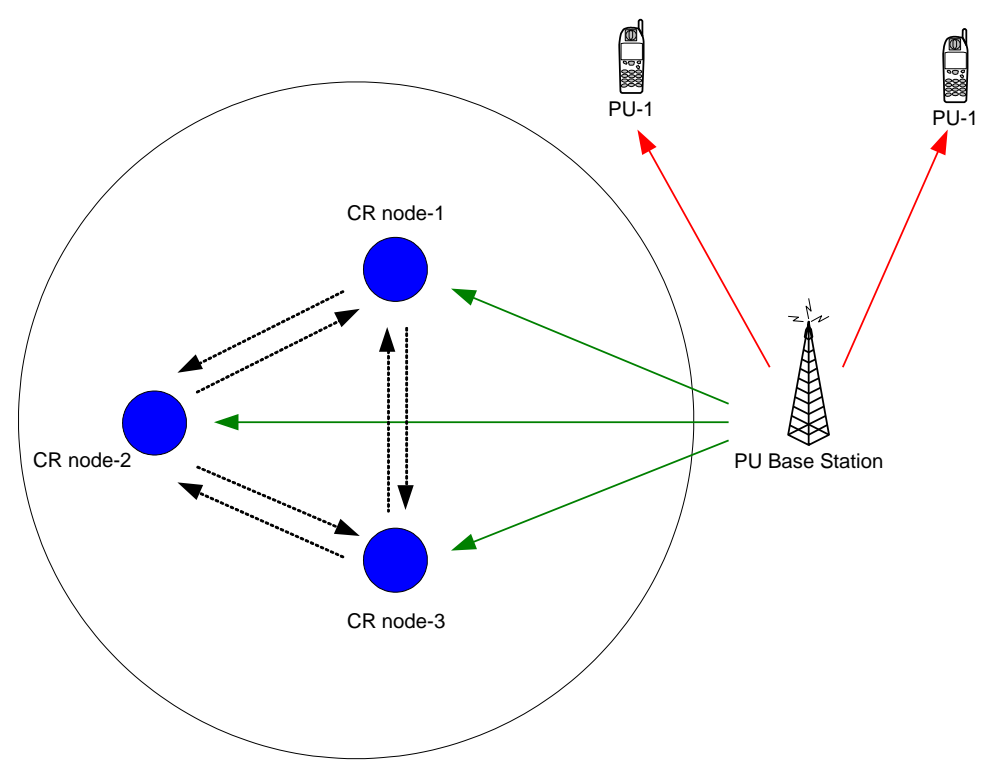

Figure 4. DMCSS model

To validate this work, multiuser decentralized cooperative performance will compare with single user sensing, multiuser random sensing and multiuser centralized cooperative sensing, where the throughput performance (bits/slot) is presented as a function of a slot number $(T)$. In single user sensing, one user without cooperation selects a channel, while random sensing assumes that each SU randomly selects a channel to sense at each time slot. The simulation set the number of $\mathrm{SU}=5$, the number of channel $\mathrm{N}=10$ with the same bandwidth $\mathrm{B}=1$, the transition probability $\alpha=0.1$ and $\beta=0.9$, the collision threshold or maximum collision allowed by PU $\zeta=0.3$, and the number of $\operatorname{slot}(\mathrm{T})=25$.

The achieved average throughput of SU is shown in Figure 5. In single user, centralized and decentralized cooperative sensing model, it can be stated that observation-based information has a significant impact to the throughput performance. Secondary user can gain the reward by improving the throughput performance during $\mathrm{T}$ slot transmission time. 
However, in decentralized multiuser random CSS model, reward cannot be improved and the curve tends to be constant relatively. In such case, SU consider that all channels have identical probability for idle and busy states, so that SU randomly senses and accesses channels. Unlike multiuser random sensing case, SU in centralized and decentralized cooperative sensing accesses channels with the highest probability for idle state.

According to the obtained results, cooperative spectrum sensing has a robust performance against single user sensing. The performance of CMCSS outperforms DMCSS model. However, DMCSS model still outperforms multiuser random CSS.

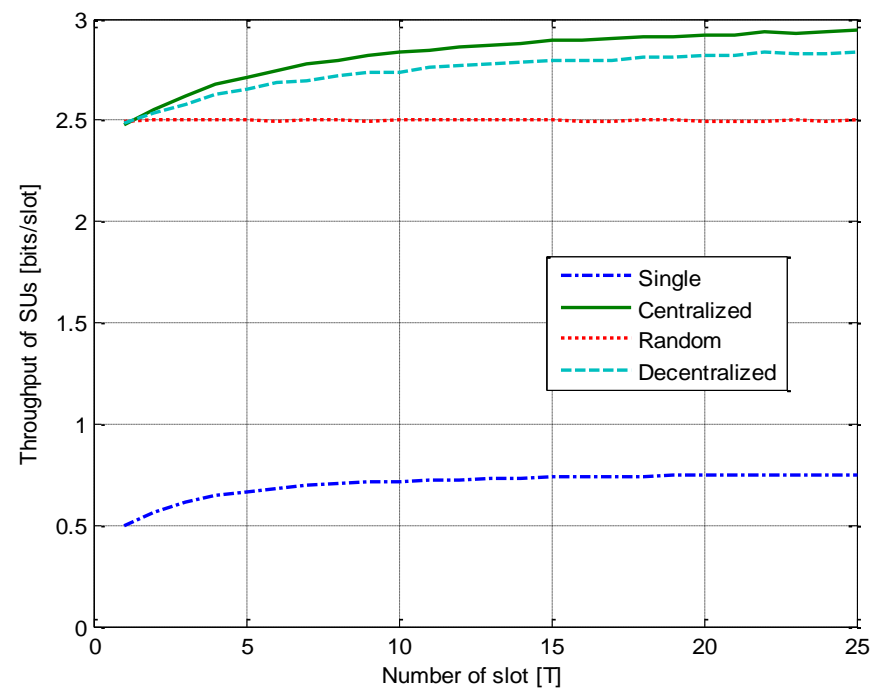

Figure. 5. Comparison between single user, centralized and decentralized cooperative user, and random sensing strategy

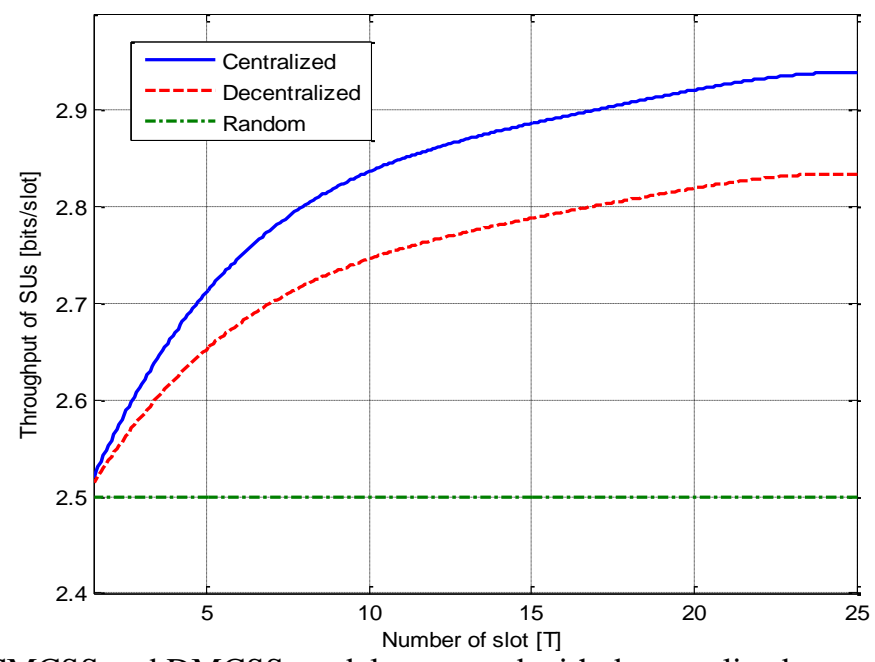

Figure. 6. CMCSS and DMCSS model compared with decentralized cooperative random sensing strategy

Figure 6 clearly shows the throughput performance for CMCSS and DMCSS model as a function of probability for selected channel (p). Single user sensing model is removed to clearly show the comparisons among each CSS model. The results show an excellent performance for CMCSS model as compared with DMCSS and multiuser random CSS. The further comparison between CMCSS and DMCSS model is shown in Figure. 7. The result 
plots throughput as a function of probability of selected channel $(p)$. Increasing $p$ affects on improving throughput of DMCSS model significantly. The throughput of DMCSS performance reaches an equal value as CMCSS model at $p=1$.

\section{Conclusion}

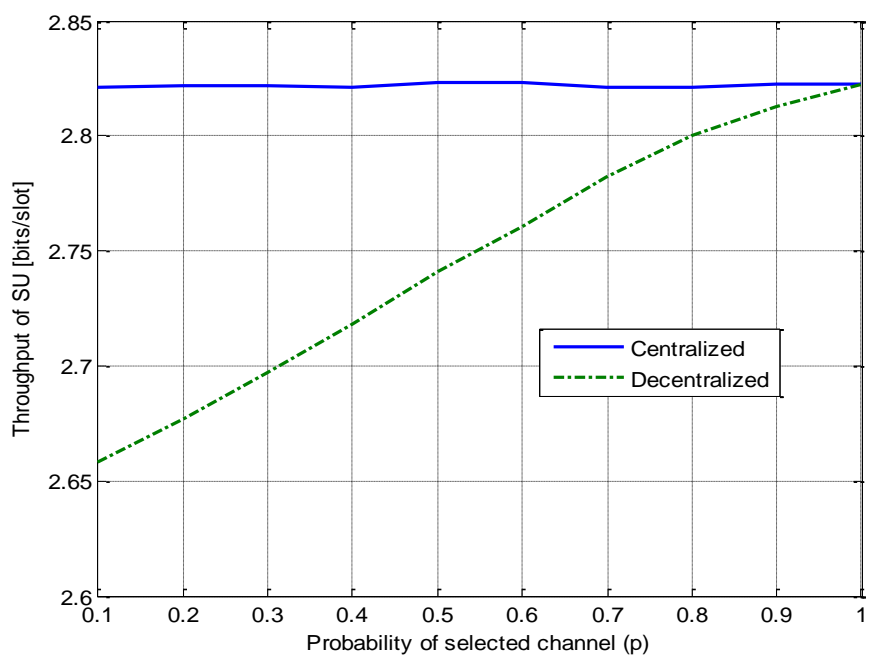

Figure. 7. CMCSS and DMCSS model as a function of $p$ (probability of selected channel)

We have studied decentralized CSS model under POMDP framework. The derived results confirmed the robustness of CSS model against single user sensing. Although CMCSS outperforms DMCSS model, however DMCSS still outperforms decentralized random CSS and single user sensing model. Probability of selected channel has a significant impact to the throughput performance. It leads to improve the throughput performance of DMCSS model. The throughput of DMCSS model reached the performance of CMCSS model at probability of selected channel $p=1$. Furthermore, exploring the strength of DMCSS method over the CMCSS one in order to find the performance-complexity trade-off is required for further study.

\section{References}

[1]. Tzamaloukas and J. Garcia-Luna-Aceves, "Channel-hopping multiple access," in IEEE ICC, June 2000.

[2]. H. Wang, H. Qin, L. Zhu, "A Survey on MAC Protocols for Opportunistic Spectrum Access in Cognitive Radio Networks," In Proc. of International Conference on Computer Science and Software Engineering, 2008.

[3]. C. Cormio, K. R. Chowdury, "A Survey on MAC Protocols for Cognitive Radio Networks," Journal of Ad Hoc Networks, pp. 1315-1329, Elsevier, 2009.

[4]. I. F. Akyildiz, W. Y. Lee, K. R. Chowdury, "CRAHNs: Cognitive Radio Ad Hoc Networks," Journal of Ad Hoc Networks, pp. 810-836, Elsevier, 2009.

[5]. K. B. Letaief and W. Zhang, "Cooperative spectrum sensing," Cognitive Wireless Communication Networks, Springer, pp. 115-138, Oct. 2007

[6]. Ghasemi, E. S. Sousa, "Opportunistic spectrum access in fading channels through collaborative sensing," In Journal of Communication, Vol. 2, No. 2, pp. 71-82, 2007.

[7]. G. Ganesan, Y. Li, "Cooperative spectrum sensing in cognitive radio, Part I: Two user networks; Part II: Multiuser networks," In IEEE Trans. On Wireless Communications, Vol. 6, No. 6, $2204-2222,2007$.

[8]. Z. Quan, S. Cui, and A. H. Sayed, "Optimum linear cooperation for spectrum sensing in cognitive radio networks," In IEEE J. Sel. Topics Signal Process, Vol. 2, No. 1, 28-40, 2008 . 
[9]. L. S. Cardoso, M. Debbah, P. Bianchi, J. Najim, "Cooperative spectrum sensing using random matrix theory," In Proc. of IEEE ISWPC, 334-338, 2008.

[10]. Pratas, N.; Marchetti, N.; Prasad, N.R.; Rodrigues, A.; Prasad, R., "Decentralized Cooperative Spectrum Sensing for Ad-Hoc Disaster Relief Network Clusters," in Vehicular Technology Conference (VTC 2010-Spring), 2010 IEEE 71st, vol., no., pp.1-5, 16-19 May 2010.

[11]. Noorshams, N.; Malboubi, M.; Bahai, A., "Centralized and decentralized cooperative spectrum sensing in cognitive radio networks: A novel approach," in Signal Processing Advances in Wireless Communications (SPAWC), 2010 IEEE Eleventh International Workshop on, vol., no., pp.1-5, 20-23 June 2010

[12]. Q. Zhao, L. Tong, A. Swami and Y. Chen, "Decentralized cognitive MAC for opportunistic spectrum access in ad hoc networks: a POMDP framework," IEEE $J$. Selected Areas Commun, pp. 589-600, 2007.

[13]. Y. Chen, Q. Zhao, A. Swami, "Joint Design and Separation Principle for Opportunistic Spectrum Access in The Presence of Sensing Errors," IEEE Transactions on Information Theory, Vol. 54, No. 5, May 2008.

[14]. Q. Zhao, S. Geirhofer, L. Tong, B. M. Sadler, "Opportunistic spectrum access via periodic channel sensing," IEEE Transaction on Signal Processing, Vol.56, No.2, Feb.2008.

[15]. S. Shetty, M. Song, C. Xin, E. K. Park, "A learning-based multiuser opportunistic spectrum access approach in unslotted primary networks," in Proc. of IEEE INFOCOM, 2009.

[16]. J. Unnikrishnan, V. V. Veeravalli, "Algorithm for dynamic spectrum access with learning for cognitive radio," IEEE Transaction on Signal Processing, Vol. 58, No. 2, Feb 2010.

[17]. Armi, N, N. M. Saad, M.Zuki.Y, M.Arshad, "Optimal Sensing for Opportunistic Spectrum Access in Cognitive Radio," International Journal of Engineering (IJE), Vol.4, Issue 3, ISSN: 1985-2312, July 2010.

[18]. Armi, N., Saad, N. M., Yusoff, M.Z., Arshad, M., "MAC protocol for opportunistic spectrum access in cognitive radio system," In Proc. of IEEE Symposium on Industrial Electronics and Applications (ISIEA), 2010.

[19]. Armi, N., Yusoff, M. Z., Saad, N. M., "Sensing and access over imperfect channel in opportunistic spectrum access system," In Proc. of National Postgraduate Conference (NPC), 2011

[20]. Armi, N., Yusoff, M. Z., Saad, N. M., "Spectrum sensing and throughput performance in opportunistic spectrum access system," In Proc. of IEEE Symposium on Wireless Technology and Application (ISWTA), 2011.

[21]. N. Armi, M. Z. Yusoff, N. M. Saad, "Multiuser Sensing for Opportunistic Spectrum Access in Cognitive Radio Networks," European Journal of Scientific Research (EJSR), Vol. 52 Issue 4, 2011.

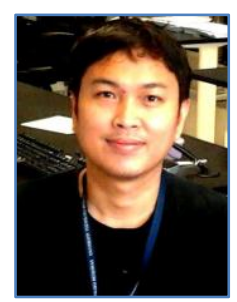

Nasrullah Armi received the B.Eng degree from National Institute of Technology, Indonesia in 1997, the M.Eng. degree from Knowledge based Information Engineering, Toyohashi University of Technology, Japan in 2004. Then, he completed Ph.D degree from Department of Electrical and Electronic Engineering, Universiti Teknologi PETRONAS, Malaysia in 2013. He joined Indonesian Institute of Sciences, Research Center for Electronics and Telecommunication in Bandung as Research Engineer in 1998. Since 2013 he has been a Senior Researcher at the same institute. He is also a senior lecturer at $\mathrm{Al}$ Aitam Foundation in Bandung. His research interest includes wireless communication, dynamic spectrum access, cognitive radio, and wireless network.

He served as a reviewer and technical program committee for a number of international conferences and designated reviewer for IEEE Transactions on Wireless Communications and 
Wireless Communication and Mobile Computing Journal. He was a student member of IEICE Japan from 2002 to 2004 and currently he is a member of IEEE, Indonesian Chapter.

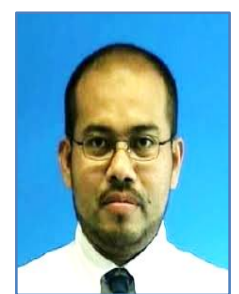

Mohd. Zuki Yusoff received the B.Sc degree from Syracuse University, Syracuse, USA, M.Sc degree from University of Surrey, Guildford, UK, and Ph.D degree from Universiti Technologi PETRONAS, Malaysia in 1988, 2001, and 2009 respectively. He was a member of Tau Beta Pi (The National Engineering Society) and Eta Kappa $\mathrm{Nu}$ (The Electrical and Computer Engineering Honorary Society). His research interests are in signal processing, communication, Embedded Systems (microprocessors and microcontrollers), networks and control. He is currently working as senior lecturer in Department of Electrical and Electronics Engineering, Universiti Technologi PETRONAS, Malaysia.

He has been supervising undergraduate and postgraduate students and published number of papers in prestigious conferences and journals. He also served as a reviewer and technical program committee for a number of international conferences and designated reviewer for journals

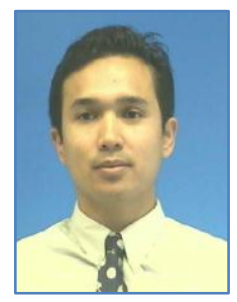

Naufal Mohamad Saad received the B.Eng degree from Institute Universitaire de Technologie(IUT) de Colmar, France, M.Eng degree from Ecole Nationale Supérieure d'Ingénieurs de Limoges, France, and Ph.D degree from Ecole Doctorale Science-Technologie-Sante, Université de Limoges, France. His research interests are in optical communication, optoelectronics, and wireless networking. He is currently working as senior lecturer in Department of Electrical and Electronics Engineering, Universiti Technologi PETRONAS, Malaysia.

He has been supervising undergraduate and postgraduate students and involved actively in IEEE membership, Malaysia chapter. He has been published number of papers in prestigious conferences and journals and served as a reviewer and technical program committee for a number of international conferences and designated reviewer for journals. 